\title{
Bifurcation of Safe Basins and Chaos in Nonlinear Vibroimpact Oscillator under Harmonic and Bounded Noise Excitations
}

\author{
Rong Haiwu, ${ }^{1}$ Wang Xiangdong, ${ }^{1}$ Luo Qizhi, ${ }^{1}$ Xu Wei, ${ }^{2}$ and Fang Tong ${ }^{2}$ \\ ${ }^{1}$ Department of Mathematics, Foshan University, Foshan 528000, China \\ ${ }^{2}$ Department of Applied Mathematics, Northwestern Polytechnical University, Xian 710072, China
}

Correspondence should be addressed to Rong Haiwu; ronghw@foshan.net

Received 10 August 2014; Revised 5 October 2014; Accepted 7 October 2014; Published 21 December 2014

Academic Editor: Qingdu Li

Copyright (C) 2014 Rong Haiwu et al. This is an open access article distributed under the Creative Commons Attribution License, which permits unrestricted use, distribution, and reproduction in any medium, provided the original work is properly cited.

\begin{abstract}
The erosion of the safe basins and chaotic motions of a nonlinear vibroimpact oscillator under both harmonic and bounded random noise is studied. Using the Melnikov method, the system's Melnikov integral is computed and the parametric threshold for chaotic motions is obtained. Using the Monte-Carlo and Runge-Kutta methods, the erosion of the safe basins is also discussed. The sudden change in the character of the stochastic safe basins when the bifurcation parameter of the system passes through a critical value may be defined as an alternative stochastic bifurcation. It is founded that random noise may destroy the integrity of the safe basins, bring forward the occurrence of the stochastic bifurcation, and make the parametric threshold for motions vary in a larger region, hence making the system become more unsafely and chaotic motions may occur more easily.
\end{abstract}

\section{Introduction}

Nonsmooth factors arise naturally in engineering applications, such as impacts, collisions, and dry frictions [1]. A considerable amount of research activities have focused on nonsmooth dynamical systems, including vibroimpact systems, collisions dynamics, chattering dynamics, and stickslip motions, in recent years. In practice, engineering structures are often subjected to time dependent loadings of stochastic nature, such as the natural phenomena due to wind gusts, earthquakes, ocean waves, and random disturbance or noise which always exists in a physical system. The influence of random excitation on the dynamical behavior of a vibroimpact dynamical system has caught the attention of many researchers. Many effective methods have been developed, for example, linearization method used by Metrikyn [2], quasistatic approach method used by Stratonovich [3], exponential polynomial fitting method proposed by Zhu [4], Markov process method used by Jing and Young [5], stochastic averaging method used by Xu et al. [6, 7], variable transformation method used by Zhuravlev [8], energy balance method used by Iourtchenko and Dimentberg [9], mean impact Poincaré map method used by Feng and He [10], path integration method used by Iourtchenko and Song [11], and numerical simulation method used by Dimentberg et al. [12] In [13], the authors tried to review and summarize the existing methods, results, and literature available for solving problems of stochastic vibroimpact systems. However, most researches focused on responses statistics, such as statistic moment and probability density function of the vibroimpact oscillator, and few are focused on the bifurcations and chaos of the stochastic vibroimpact dynamical systems.

It is well known that, by calculating the distance between the stable and unstable manifold, Melnikov's method [14] gives a powerful approximate tool for investigating chaotic motions in deterministic smooth system. However, classical Melnikov's method is not directly appropriate in the nonsmooth system. Some effective Melnikov's methods have been proposed for deterministic piecewise smooth dynamical systems $[15,16]$ and nonlinear vibroimpact dynamical systems [17]. To our knowledge, few Melnikov's methods are well developed for the stochastic vibroimpact dynamical systems.

In this paper, the bifurcation of safe basins and chaos of a nonlinear vibroimpact oscillator under both harmonic and bounded noise excitation are investigated. The impact considered here is an instantaneous impact with restitution factor $e$. The paper is organized as follows. In Section 2, Melnikov's method is extended to the analysis of homoclinic 
bifurcation in the stochastic vibroimpact system, and the conditions for the onset of chaos are derived in the mean square value sense. In Section 3, the variations of safe basins are presented numerically when one changes the amplitude of the harmonic excitation both in the deterministic and stochastic cases. Conclusions are presented in Section 4.

\section{The Stochastic Melnikov Function}

2.1. Theoretical Analysis. Consider a classical Duffing vibroimpact oscillator with bilateral constrains under both harmonic and bounded random noise excitations governed by the following equation:

$$
\begin{gathered}
\ddot{x}-x+\alpha x^{3}=\varepsilon\left(-\beta \dot{x}+f_{1} \cos \omega_{1} t+f_{2} \xi(t)\right), \\
|x|<1, \\
\dot{x}_{+}=-(1-\varepsilon \rho) \dot{x}_{-}, \quad|x|=1,
\end{gathered}
$$

where the dot indicates differentiation with respect to time $t, \varepsilon$ is a small scale, $0<\varepsilon \ll 1, \alpha$ represents the intensity of the nonlinear term, $\beta$ is the damping coefficient, $f_{1}$ and $\omega_{1}$ are amplitude and frequency of the deterministic excitation, respectively, and $0<e \leq 1$ is the restitution factor to be a known parameter of impact losses, $e=1-$ $\varepsilon \rho$, whereas subscripts "minus" and "plus" refer to value of response velocity just before and after the instantaneous impact. Thus $\dot{x}_{+}$and $\dot{x}_{-}$are actually rebound and impact velocities of the mass, respectively. They have the same magnitude whenever $e=1$; therefore this special case is that of elastic impacts, whereas in case $e<1$ some impact losses are observed. $f_{2}$ denotes the intensity of the random excitation and $\xi(t)$ is a bounded noise process, which was introduced by Stratonovich [18]:

$$
\xi(t)=\cos \left(\omega_{2} t+\sigma W(t)+\theta\right),
$$

where $\omega_{2}>0$ is the frequency of the random excitation, $W(t)$ is a standard Wiener process, and $\theta$ a uniformly distributed random number in $(0,2 \pi) . \sigma \dot{W}(t)$ is the random disorder which describes random temporal deviations of the excitation frequency from its expectation or mean $\omega_{2}$. The process $\xi(t)$ has the following power spectral density [19]:

$$
S(\omega)=\frac{\sigma^{2}}{2 \pi}\left[\frac{1}{4\left(\omega-\omega_{2}\right)^{2}+\sigma^{4}}+\frac{1}{4\left(\omega+\omega_{2}\right)^{2}+\sigma^{4}}\right] .
$$

Obviously $|\xi(t)| \leq f_{2}$ is a bounded random process. Periodicin-time excitation with a "random disorder," or random phase modulation appears in structural dynamics with traveling loadings and/or structures, having certain imperfect spatial periodicity in certain problems of aeroelasticity [19]. This process will be assumed to be narrow-band, which is clearly seen to be in the case provided that $\sigma \rightarrow 0$. We assume that $\sigma \ll \omega_{2}$ in this paper. The bounded noise model (2) is a suitable model and many researches have been done on the responses of nonlinear system under bounded noise excitation $[20,21]$. Obviously, bounded noise $\xi(t)$ has continuous sample function; hence Melnikov's method may be used in the stochastic system (1).

The physical model of (1) can be viewed as the motion of a mass with harden stiffness under both harmonic and bounded random noise external excitations, while two collision obstacles are placed before and after the equilibrium position with distance 1 .

When $\varepsilon=0$, system (1) reduces to an unperturbed deterministic vibroimpact system. Using stability analysis, one collects two center-type fixed points $(\sqrt{1 / \alpha}, 0)$ and $(-\sqrt{1 / \alpha}, 0)$, and a saddle-type fixed point $(0,0)$. Denotes $y=$ $\dot{x}$; two homoclinic orbits connecting the two center-type fixed points and saddle-type fixed point are

$$
\begin{array}{r}
\left(x_{u}, y_{u}\right)=\left( \pm \sqrt{\frac{2}{\alpha}} \operatorname{sech}\left(t-t_{0}\right),\right. \\
\left.\mp \sqrt{\frac{2}{\alpha}} \operatorname{sech}\left(t-t_{0}\right) \tanh \left(t-t_{0}\right)\right), \\
\quad t \leq 0, \\
\left(x_{s}, y_{s}\right)=\left( \pm \sqrt{\frac{2}{\alpha}} \operatorname{sech}\left(t+t_{0}\right),\right. \\
\left.\mp \sqrt{\frac{2}{\alpha}} \operatorname{sech}\left(t+t_{0}\right) \tanh \left(t+t_{0}\right)\right), \\
\quad t \geq 0,
\end{array}
$$

where $t_{0}=\operatorname{arcsech}(\sqrt{\alpha / 2})$.

Equation (4) is exactly the solution of the unperturbed deterministic system (1) as $\varepsilon=0$ without vibroimpact, and similar system has been discussed in [17] by using the Melnikov method. Using the results in $[17,22]$, one obtains the stochastic Melnikov function for homoclinic orbits of system (1):

$$
\begin{aligned}
M(\theta)= & \rho M_{d 1}(\theta)+\beta M_{d 2}(\theta) \\
& +f_{1} M_{d 3}(\theta)+f_{2} M_{r}(\theta),
\end{aligned}
$$

where $M_{d 1}(\theta), M_{d 2}(\theta)$, and $M_{d 3}(\theta)$ are the deterministic parts of the random process $M(\theta)$; they are caused by the deterministic harmonic excitation, and $M_{r}(\theta)$ represents the stochastic term which is caused by the bounded noise $\xi(t)$ :

$$
\begin{aligned}
M_{d 1}(\theta)= & \frac{2}{\sqrt{1-(\alpha / 2)}} \int_{0}^{1}\left(x-\alpha x^{3}\right) d x=-2 \sqrt{1-\frac{\alpha}{2}} \\
M_{d 2}(\theta)= & -\frac{1}{\sqrt{1-(\alpha / 2)}}\left[\int_{-\infty}^{0} y_{u}^{2}(t) d t+\int_{0}^{+\infty} y_{s}^{2}(t) d t\right] \\
= & -\frac{2}{\alpha \sqrt{1-(\alpha / 2)}} \\
& \times\left[\int_{-\infty}^{0} \operatorname{sech}^{2}\left(t-t_{0}\right) \tanh ^{2}\left(t-t_{0}\right) d t\right. \\
& \left.\quad+\int_{0}^{+\infty} \operatorname{sech}^{2}\left(t+t_{0}\right) \tanh ^{2}\left(t+t_{0}\right) d t\right]
\end{aligned}
$$




$$
\begin{aligned}
&=-\frac{4}{3 \alpha \sqrt{1-(\alpha / 2)}}\left(1-\tanh ^{3} t_{0}\right) \\
&=-\frac{4}{3 \alpha \sqrt{1-(\alpha / 2)}}\left[1-\left(1-\frac{\alpha}{2}\right)^{3 / 2}\right], \\
& M_{d 3}(\theta)= \frac{1}{\sqrt{1-(\alpha / 2)}}\left[\int_{-\infty}^{0} \cos \left(\omega_{1} t+\omega_{1} \theta\right) y_{u}(t) d t\right. \\
&=\left.-\frac{1}{\sqrt{(2 / \alpha)-1}} \cos \left(\omega_{1} t+\omega_{1} \theta\right) y_{s}(t) d t\right] \\
& \times {\left[\int_{-\infty}^{-t_{0}} \cos \left(\omega_{1} t+\omega_{1} \theta+\omega_{1} t_{0}\right) \operatorname{sech}(t) \tanh (t) d t\right.} \\
&\left.+\int_{t_{0}}^{+\infty} \cos \left(\omega_{1} t+\omega_{1} \theta-\omega_{1} t_{0}\right) \operatorname{sech}(t) \tanh (t) d t\right] \\
&=-\frac{1}{\sqrt{(2 / \alpha)-1}} \sin \left(\omega_{1} \theta\right) \\
& \times \int_{t_{0}}^{+\infty} \sin \left(\omega_{1} t-\omega_{1} t_{0}\right) \operatorname{sech}(t) \tanh (t) d t, \\
& M_{r}(\theta)=\frac{1}{\sqrt{1-(\alpha / 2)}} \\
& \times \int_{-\infty}^{0} y_{u}(t) \xi(t+\theta) d t \\
&\left.y_{s}(t) \xi(t+\theta) d t\right] .
\end{aligned}
$$

According to the dynamic theory, the stable and the unstable manifolds will intersect transversely with each other which means chaos will occur when there exist simple zeros in Melnikov function (5) in the deterministic case $f_{2}=0$. However, in the stochastic case $f_{2} \neq 0$, the Melnikov function measures the random distance between the stable and the unstable manifolds. In this case, the threshold value for the rising of the chaotic motion depends on the property of the random excitation process and may deviate from the one for the deterministic case. In order to analyze the simple zero points of $M(\theta)$ in the statistics sense, one considers the following equations:

$$
\begin{gathered}
E[M(\theta)-E[M(\theta)]]^{2}=E\left[f_{2} M_{r}(\theta)\right]^{2}=f_{2}^{2} \sigma_{M_{r}}^{2}, \\
E[0-E[M(\theta)]]^{2}=E[M(\theta)]^{2},
\end{gathered}
$$

where $E$ represents the mathematics expectation. Since $E\left[M_{r}(\theta)\right]=0$, the condition for the onset of chaotic motion in the mean square value sense is

$$
\begin{aligned}
f_{2}^{2} \sigma_{M_{r}}^{2} & =E^{2}[M(\theta)] \\
& =\left(\rho M_{d 1}(\theta)+\beta M_{d 2}(\theta)+f_{1} M_{d 3}(\theta)\right)^{2},
\end{aligned}
$$

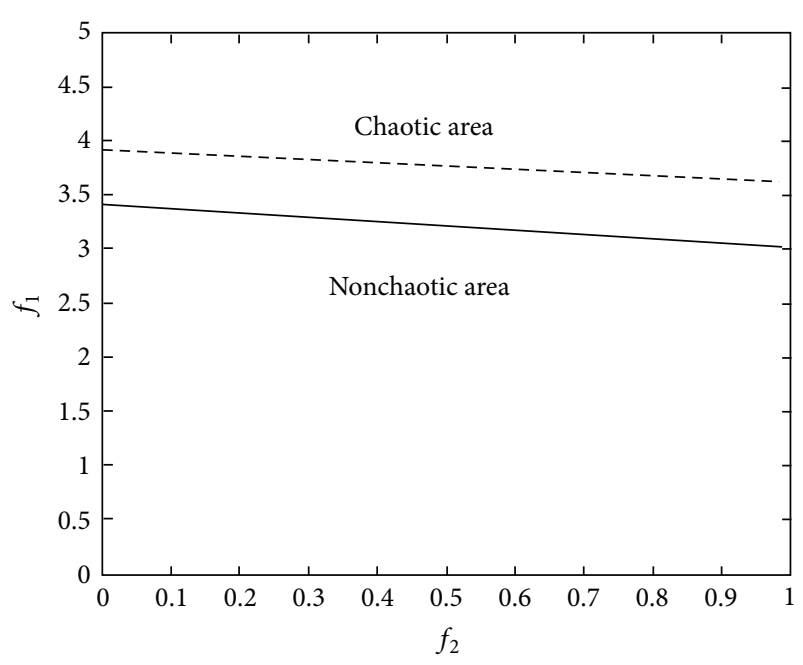

Figure 1: Chaotic area of system (1), - : theoretical solution and --- : numerical solution.

where

$$
\begin{aligned}
\sigma_{M_{r}}^{2}= & \int_{-\infty}^{+\infty}|H(\omega)|^{2} S_{\xi}(\omega) d \omega \\
= & \frac{\sigma^{2}}{2 \pi} \int_{-\infty}^{+\infty}|H(\omega)|^{2}\left[\frac{1}{4\left(\omega-\omega_{2}\right)^{2}+\sigma^{4}}\right. \\
& \left.+\frac{1}{4\left(\omega+\omega_{2}\right)^{2}+\sigma^{4}}\right] d \omega, \\
H(\omega)= & \int_{-\infty}^{0} y_{u}(t) \exp (-i \omega t) d t+\int_{0}^{+\infty} y_{s}(t) \exp (-i \omega t) d t \\
= & -\int_{-\infty}^{0} \sqrt{\frac{2}{\alpha}} \operatorname{sech}\left(t-t_{0}\right) \tanh \left(t-t_{0}\right) \exp (-i \omega t) d t \\
& -\int_{0}^{+\infty} \sqrt{\frac{2}{\alpha}} \operatorname{sech}\left(t+t_{0}\right) \tanh \left(t+t_{0}\right) \exp (-i \omega t) d t \\
= & -2 i \sqrt{\frac{2}{\alpha}} \int_{t_{0}}^{+\infty} \operatorname{sech}(t) \tanh (t) \sin \left(\omega t-\omega t_{0}\right) d t .
\end{aligned}
$$

Then, from (8), the condition of the occurrence of chaotic motions of system (1) is

$$
f_{1}>\bar{f}_{1}=\frac{\rho M_{d 1}(\theta)+\beta M_{d 2}(\theta)+f_{2} \sigma_{M_{r}}}{M_{d 3}(\theta)} .
$$

2.2. Numerical Results. Now we give some numerical results to verify the analytic conditions given by (8) and (10). The parameters of system (1) are given by

$$
\begin{gathered}
\alpha=1.4, \quad \beta=2.4, \quad \varepsilon=0.1, \quad \rho=1.8, \\
\omega_{1}=1.5, \quad \omega_{2}=1.0, \quad \sigma=0.4 .
\end{gathered}
$$

The variation of the threshold value $f_{1}$ versus the bounded noise amplitude $f_{2}$ is plotted in Figure 1 for the onset of 


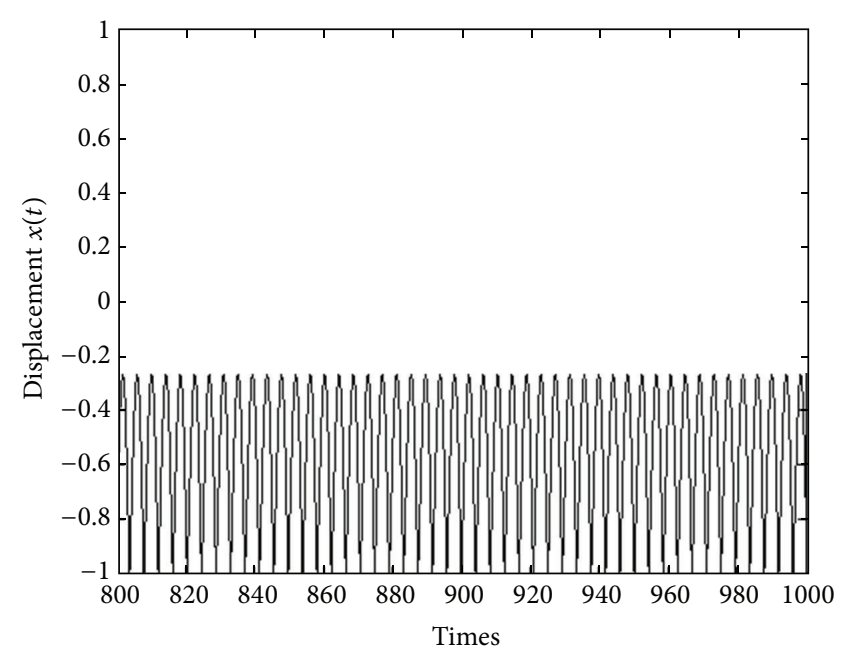

(a) Time history of $y(t)$

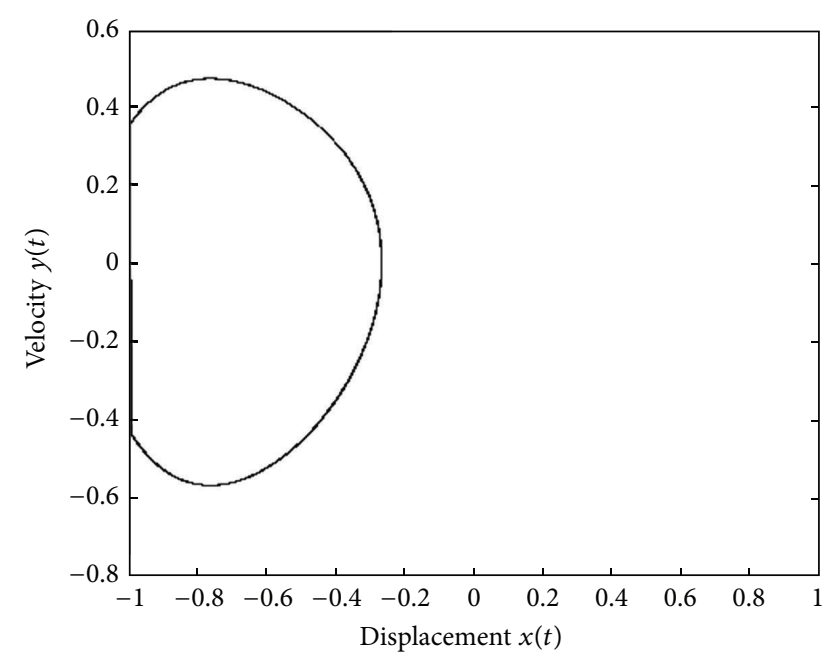

(b) Phase plot

Figure 2: Numerical results of $(1)\left(f_{1}=2.5, f_{2}=0.0, x(0)=-0.8, \dot{x}(0)=-2.5\right)$.

chaotic monition in system (1), the solid line represents the analytic results given by (10), and the dashed line represents the numerical simulations according the criterion of the largest Lyapunov exponent using the algorithm presented by Wolf et al. [23]. Usually, calculating the Lyapunov exponents is regarded as the simplest method to verify the existence of chaotic behavior. However, the Melnikov method and the erosion of the safe basins are mainly discussed in this paper, while the largest Lyapunov exponent is only used to verify the efficiency of the above two methods. The largest Lyapunov exponent of system (1) is positive in the area above the dashed line, which means the occurrence of the chaotic motion, while the largest Lyapunov exponent of system (1) is negative in the area below the dashed line, which means no occurrence of the chaotic motion. It can be seen clearly from Figure 1 that Melnikov's condition (10) is only a necessary condition for the occurrence of chaotic motion in the Lyapunov sense. It can also be seen that the threshold value $f_{1}$ will decrease when $f_{2}$ increase thus make the chaotic motions occur more easily.

Three different simulation points in Figure 1 are chosen to compare the analytic results with the numerical results. One point is $\left(f_{1}, f_{2}\right)=(2.5,0.0)$ in the deterministic case, which is below the analytic critical value $f_{1}=3.41$, Melnikov's criterion implies that the motion of system (1) is regular, and numerical simulations show that there are two coexisting attractors with the same largest exponent -0.351 . The corresponding phase portraits and time history portraits are shown in Figures 2 and 3 for different initial values. The response time history of system (1) and the phase plot are shown in Figure 2 in the case $x(0)=-0.8, \dot{x}(0)=-2.5$. Clearly, the response is a period one while the phase trajectory is a limit cycle.

The response time history of system (1) and the phase plot are shown in Figure 3 in the case $x(0)=-0.4, \dot{x}(0)=2.5$.

Figures 2 and 3 show that the stable steady state solution of a deterministic vibroimpact system may be different for different initial values; such interesting phenomenon also exists for stochastic vibroimpact system. Another simulation point is $\left(f_{1}, f_{2}\right)=(2.5,0.05)$ in the stochastic case, which is also below the analytic critical value $f_{1}=3.38$, and the largest Lyapunov exponent is -0.337 . The corresponding phase portraits and time history portraits are shown in Figures 4 and 5 for different initial values. The response time history of system (1) and the phase plot are shown in Figure 4 in the case $x(0)=-0.8, \dot{x}(0)=-2.5$. Clearly, the response is a quasiperiod one while the phase trajectory is a diffused limit cycle.

The response time history of system (1) and the phase plot are shown in Figure 5 in the case $x(0)=-0.4, \dot{x}(0)=2.5$.

Figures 4 and 5 show that the stationary response of system (1) may be different for different initial values in some parameter area. The random noise $\xi(t)$ will change the steady-state response of system (1) from a period solution to a quasiperiod solution. In fact, due to the existence of random noise, the motion may jump from one stable position to another with some, maybe small, probability. However, it may take a long time to observe such phenomenon, but the simulation time is always limited in the practical numerical simulation, so one may give the conclusion only base on finite time observed phenomenon.

It is well known, from the theory of nonlinear oscillation, that if an oscillator with hardening nonlinear stiffness is subjected to sinusoidal excitation, the response may exhibit sharp jumps in amplitude. This jump behavior is associated with the fact that, over a range of the values of the ratio of excitation frequency to the natural frequency of the degenerated linear oscillator, the response amplitude is triplevalued. Therefore the system should have two stationary responses which depend on the initial condition. However, it is a disputable problem whether there are more than one stationary response if an oscillator with hardening nonlinear stiffness is subjected to random excitations [24]. Here, it is found that there are more than one stationary response in a nonlinear stochastic vibroimpact system. 


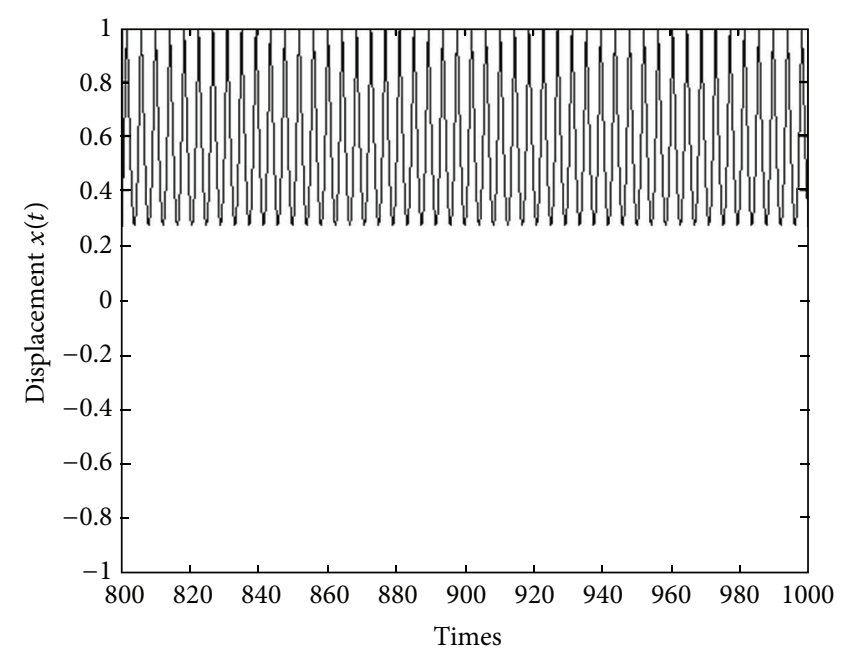

(a) Time history of $y(t)$

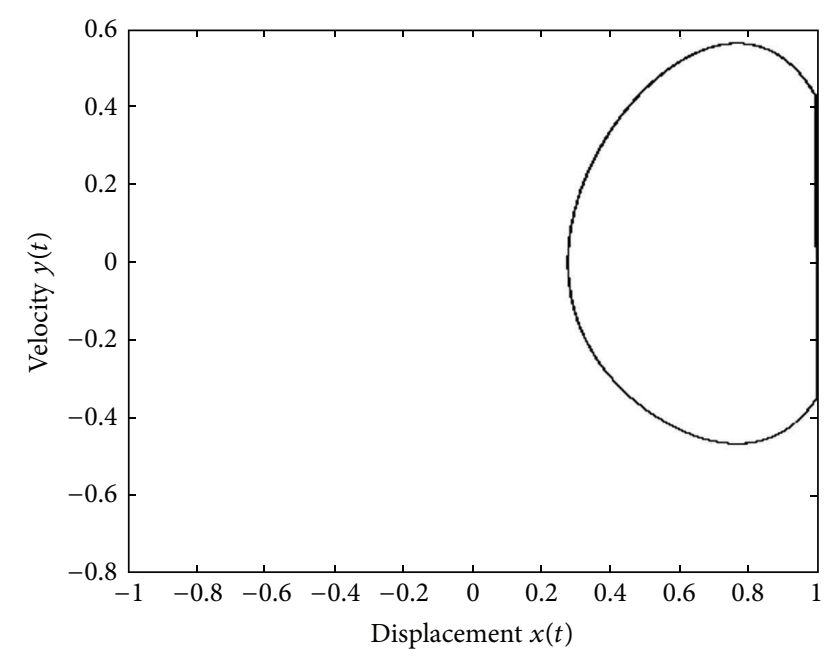

(b) Phase plot

Figure 3: Numerical results of $(1)\left(f_{1}=2.5, f_{2}=0.0, x(0)=-0.4, \dot{x}(0)=2.5\right)$.

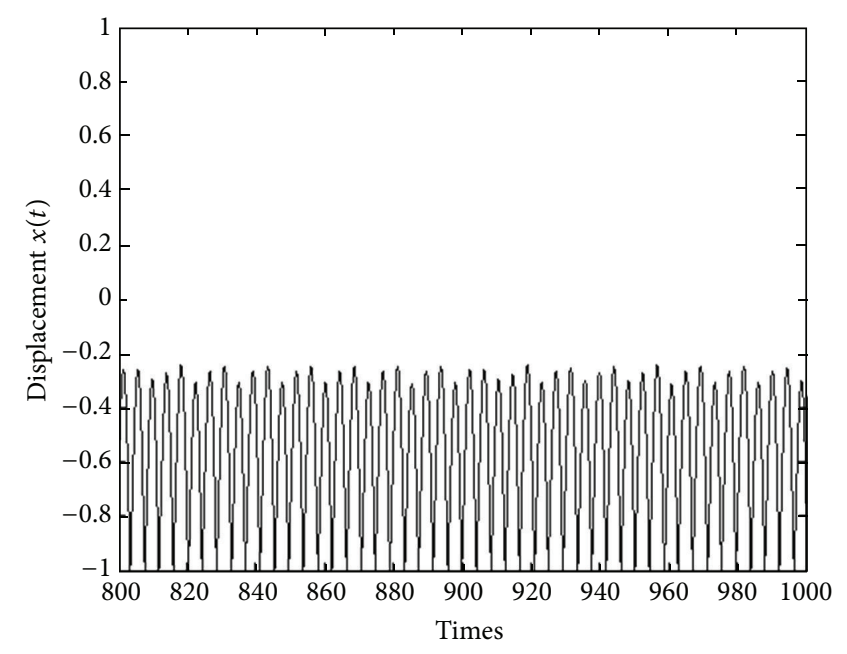

(a) Time history of $y(t)$

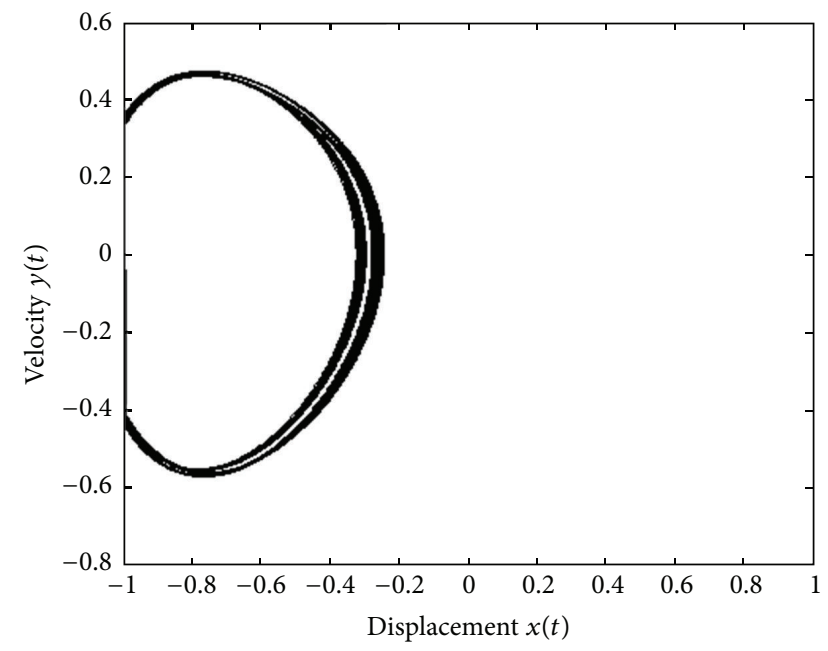

(b) Phase plot

Figure 4: Numerical results of (1) $\left(f_{1}=2.5, f_{2}=0.05, x(0)=-0.8, \dot{x}(0)=-2.5\right)$.

The third simulation point is $\left(f_{1}, f_{2}\right)=(5.5,0.05)$ in the stochastic case, which is above the analytic critical value $f_{1}=$ 3.38, the largest Lyapunov exponent is 0.062 . The response time history of system (1) and the phase plot are shown in Figure 6 in the case $x(0)=-0.8, \dot{x}(0)=-2.5$. Clearly, the response is a chaotic one.

\section{Bifurcation of Safe Basins and Chaos}

Alternative to the Melnikov function and Lyapunov exponent method, there is another method to identify the rising of chaos. One of these is to determine the global structures of the system and one of these global structures is the boundary of safe basin. The safe basin boundaries of attractors are usually fractal and naturally incursive since the coexistence of period and chaotic attractors. They are related to homoclinic or heteroclinic intersections of stable and unstable manifolds of the saddle points in the system and chaos often arises in such system. The decrease of the safe basin's area is called basin erosion and will be discussed in this section.

In some time the limitation of the vibration amplitude may be more important, since the structure of the system will be destroyed when the amplitude of the vibration passes through a critical value and thus leads to the researches of the safe basins $[25,26]$. There are some relations between erosion of safe basins and chaotic motions of the system. When the safe basin is eroded, the boundary of the sage basin will have fractal structures, and the motions initial from some points 


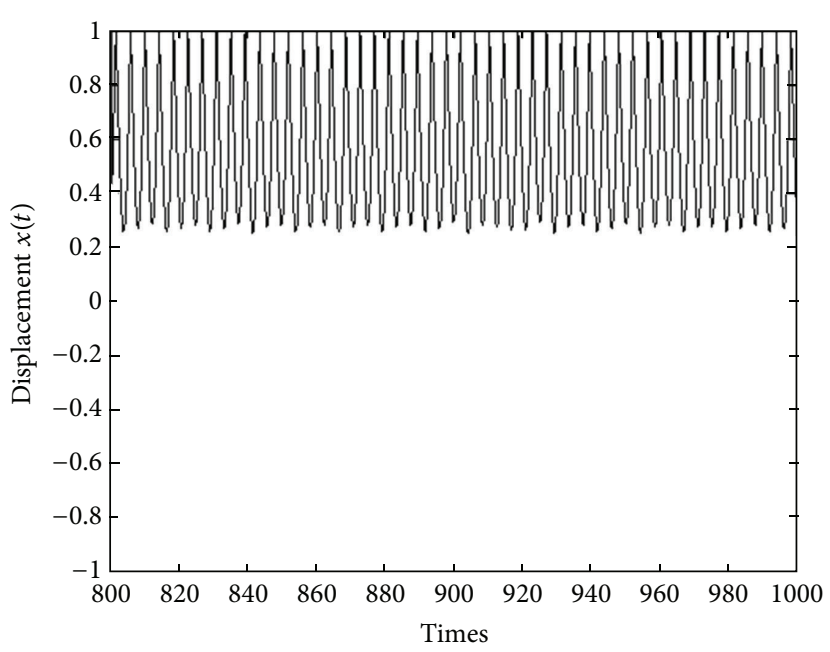

(a) Time history of $y(t)$

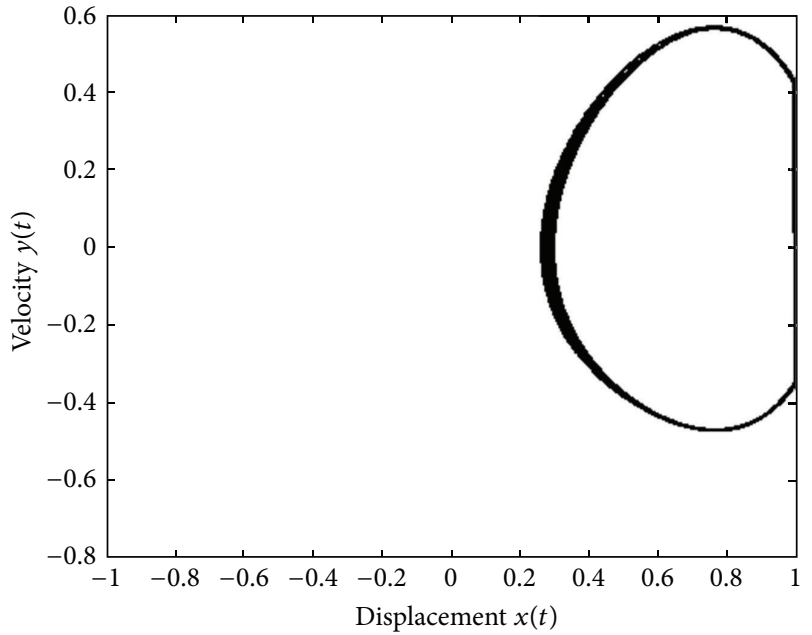

(b) Phase plot

FIGURE 5: Numerical results of $(1)\left(f_{1}=2.5, f_{2}=0.05, x(0)=-0.4, \dot{x}(0)=2.5\right)$.

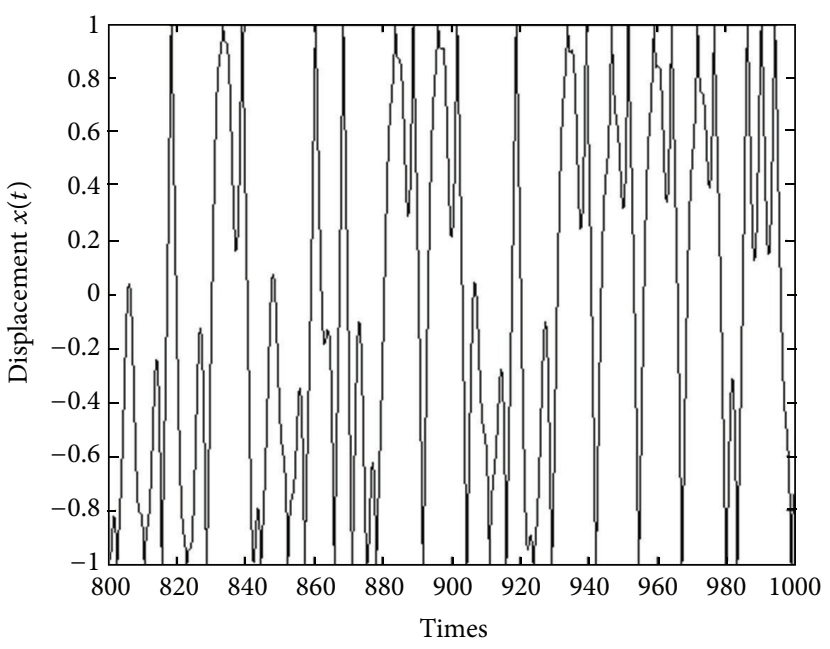

(a) Time history of $y(t)$

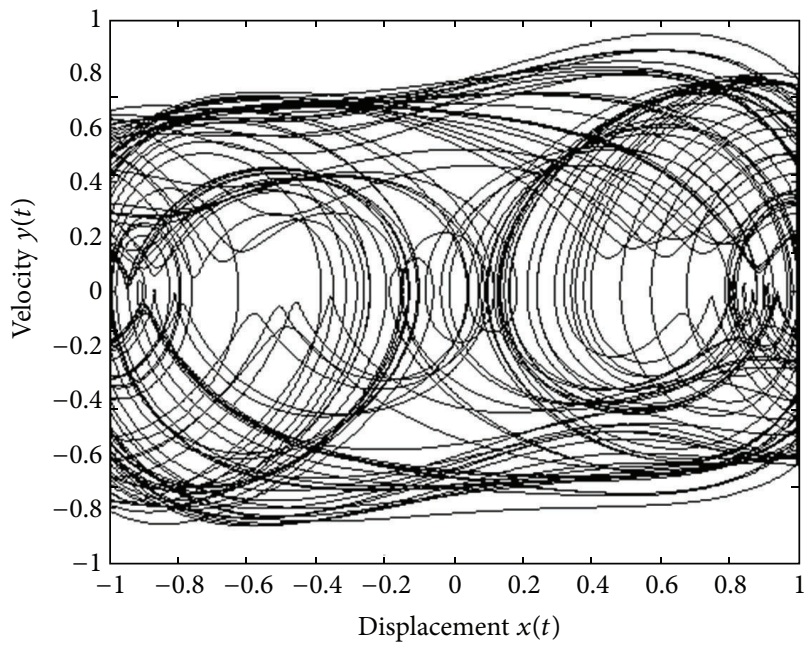

(b) phase plot

Figure 6: Numerical results of (1) $\left(f_{1}=5.5, f_{2}=0.05, x(0)=-0.8, \dot{x}(0)=-2.5\right)$.

within the safe basin will be chaotic. According to $[25,26]$, the safe basins of the system may be defined using a bounded area $D$ in the space of phase trajectories. The trajectory starting from the safe basins will be stay in the area $D$ when the time $t$ tends to infinity. Otherwise, the trajectory starting beyond the safe basins will escape the area $D$; such trajectory is unstable and may destroy or collapse the system. The structure of the safe basins is similar to some attractor basins. The acreage and shape of the safe basins will change when the parameter of the system changes.

3.1. Bifurcation without Bounded Noises. In this paper, the evolution of the safe basins of system (1) is studied numerically when the parameter $f_{1}$ changes its value in the deterministic case $f_{2}=0$, firstly. In the numerical simulation, the parameters in system (1) are chosen as:

$$
\begin{array}{rrr}
\alpha=0.8, & \beta=0.9, \quad \varepsilon=0.1, \quad \rho=1.8, \\
\omega_{1}=1.5, \quad \omega_{2}=1.0, & \sigma=0.4, \quad f_{2}=0 .
\end{array}
$$

The bounded area $D$ is defined as follows:

$$
D=\{(x, y):-1 \leq x \leq 1,-0.8 \leq y \leq 0.8\} ;
$$

then $D$ is divided into $100 \times 100$ lattices and the lattice points are taken as the initial values for the solutions of system (1). If the solution of system (1) stays in area $D$ for a long enough time up to $t=2000$, such a solution can be approximately 


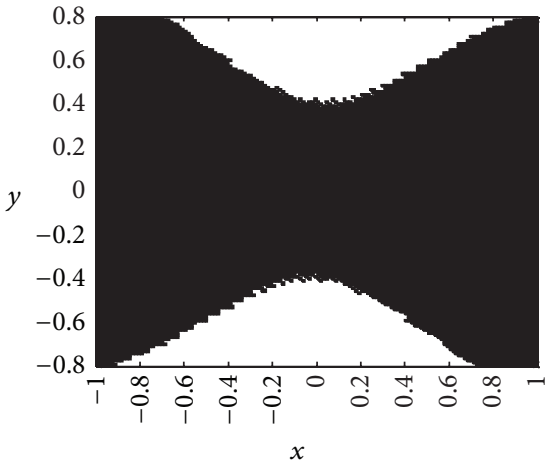

(a) $f_{1}=0.00, f_{2}=0.00, p=0.7654$

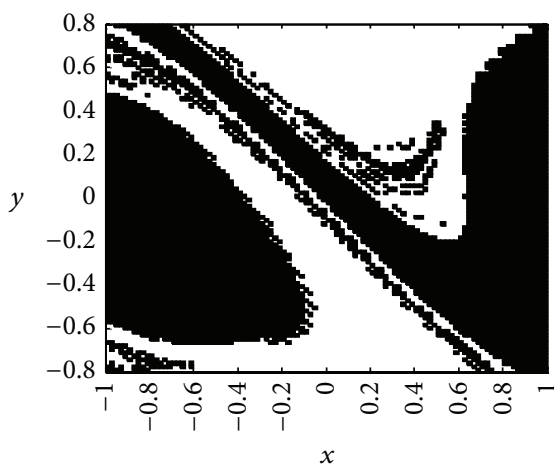

(d) $f_{1}=3.00, f_{2}=0.00, p=0.5500$

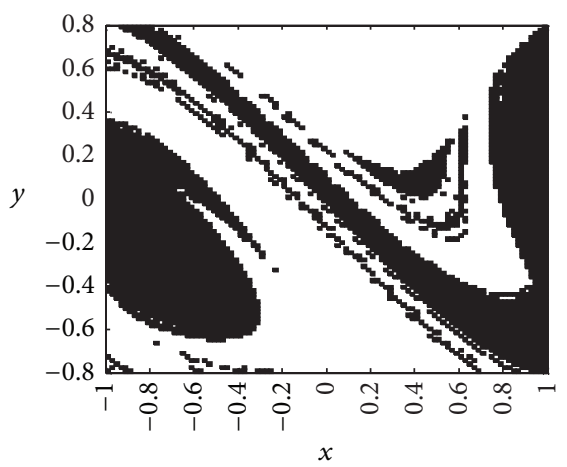

(g) $f_{1}=4.30, f_{2}=0.00, p=0.3937$

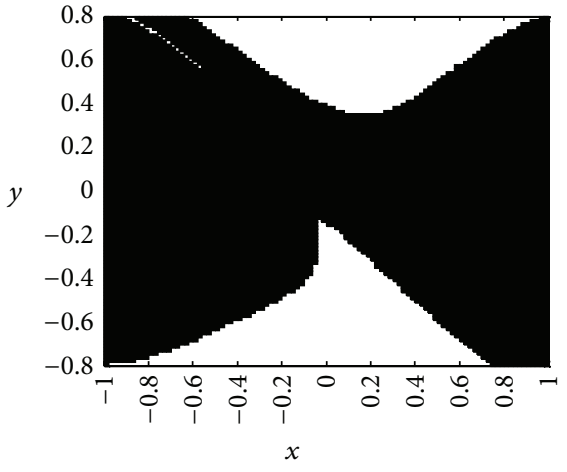

(b) $f_{1}=1.00, f_{2}=0.00, p=0.7500$

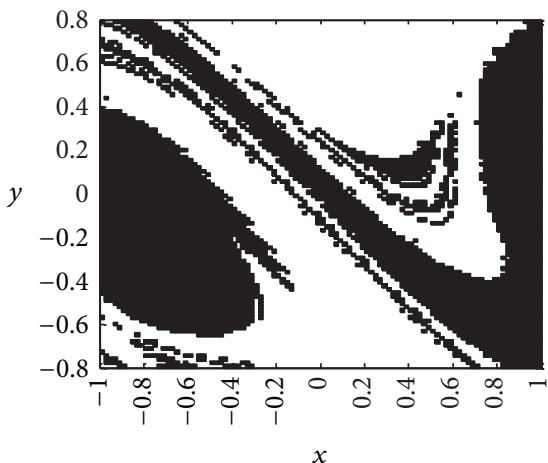

(e) $f_{1}=4.00, f_{2}=0.00, p=0.4450$

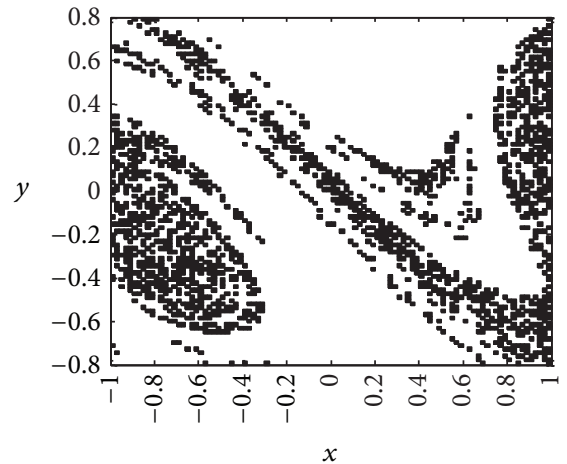

(h) $f_{1}=4.50, f_{2}=0.00, p=0.1776$

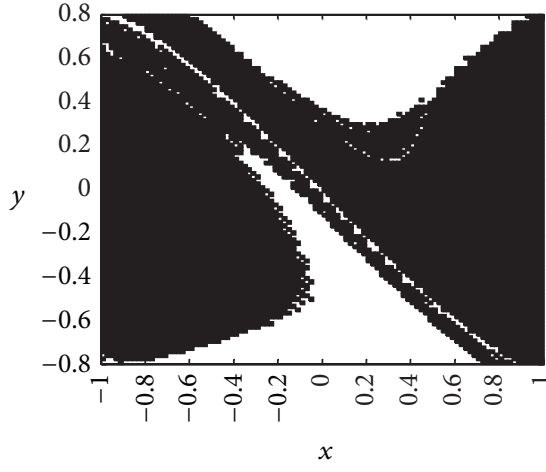

(c) $f_{1}=2.00, f_{2}=0.00, p=0.7008$

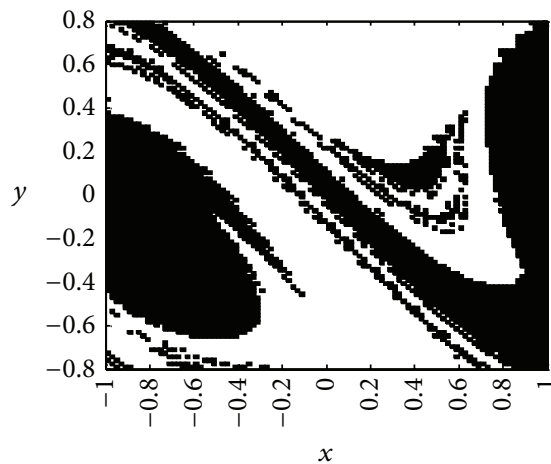

(f) $f_{1}=4.20, f_{2}=0.00, p=0.4199$

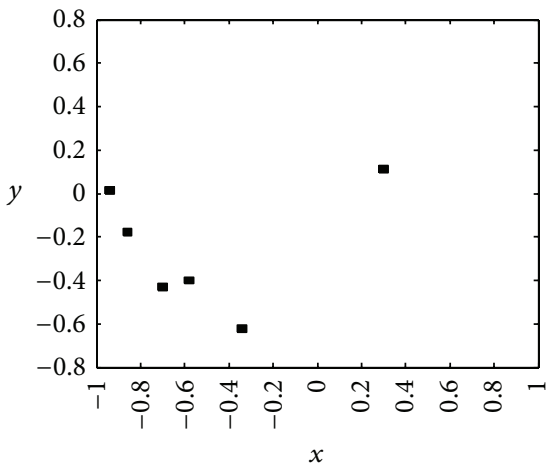

(i) $f_{1}=4.53, f_{2}=0.00, p=0.0024$

Figure 7: Erosion of safe basins in system (1): $\left(f_{2}=0\right)$.

taken as a safe solution, and the corresponding lattice may be taken as part of the safe basins; if the solution of system (1) escapes the area $D$, such a solution is taken as an unsafe solution, and the corresponding lattice is beyond the safe basins. Governing equation (1) is numerically integrated by the fourth-order Runge-Kutta algorithm, and the numerical results are shown in Figure 7(a) to Figure 7(i). The black region denotes the safe basins while the blank region represents the unsafe area in Figures 7 and 8.

The safe basins shown in Figure 7(a) are a densely packed, integral one, while the safe basins shown in Figure 7(b) to Figure 7(i) are eroded ones. Calculation results show that in the case when $f_{1} \leq f_{s}=0.21$, the boundary of the safe basins of system (1) are smooth without any erosion as shown in Figure $7(\mathrm{a})$, where $p=0.7654$ is a proportionality coefficient of the safe basins acreage to the whole acreage of area $D$. It can be seen from Figure 7 that $p$ will decease when $f_{1}$ increase which implies erosion of the safe basins. In the case when $f_{1}>f_{s}$, the boundary of the safe basins are eroded more and more with increase of $f_{1}$ as shown in Figure 7(b) to Figure 7(i), and in the case when $f_{1}>f_{e}=$ 4.54 , the safe basins disappear completely. One may call such phenomena that happen in the sudden change of the safe basins, which become from integrated one to eroded one or from eroded one to nothingness when $f_{1}$ passes through the critical values $f_{s}=0.21$ and $f_{e}=4.54$, as deterministic safe basins bifurcation. Then $f_{s}=0.21$ and $f_{e}=4.54$ are two bifurcation points if $f_{1}$ is chosen as bifurcation parameter. In 


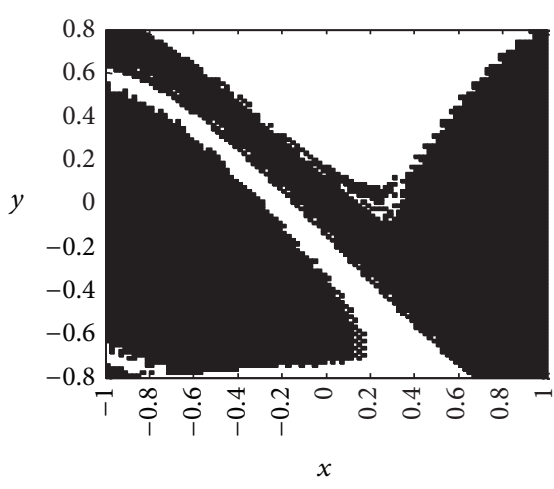

(a) $f_{1}=0.0, f_{2}=2.0, p=0.6712$

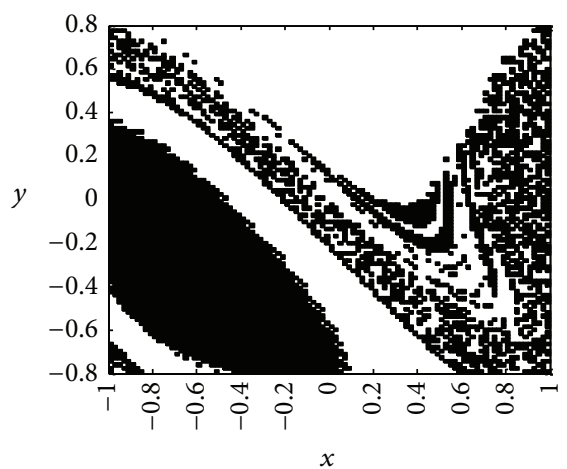

(d) $f_{1}=3.0, f_{2}=2.0, p=0.4113$

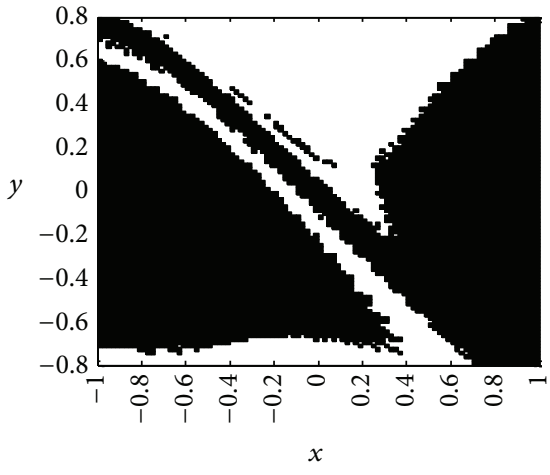

(b) $f_{1}=1.0, f_{2}=2.0, p=0.6704$

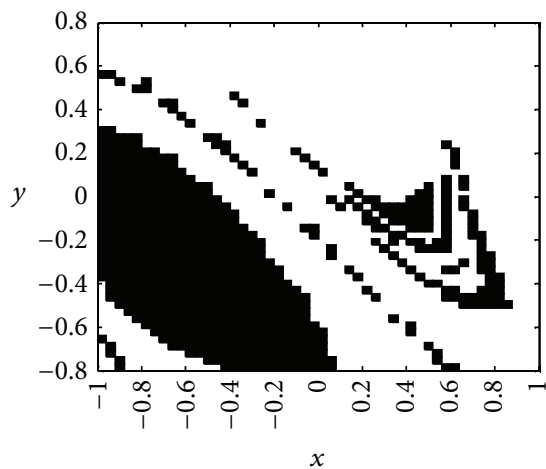

(e) $f_{1}=3.2, f_{2}=2.0, p=0.2852$

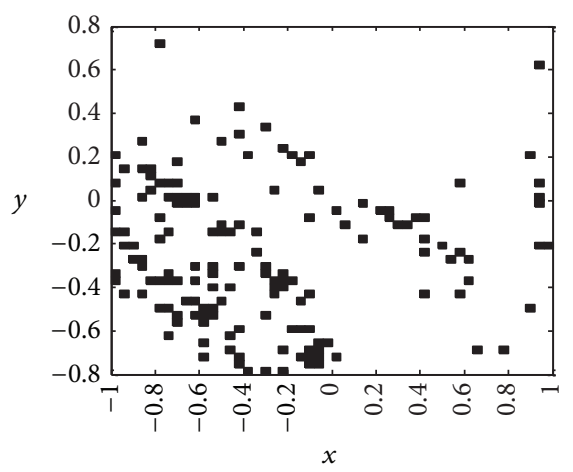

(g) $f_{1}=3.4, f_{2}=2.0, p=0.0600$

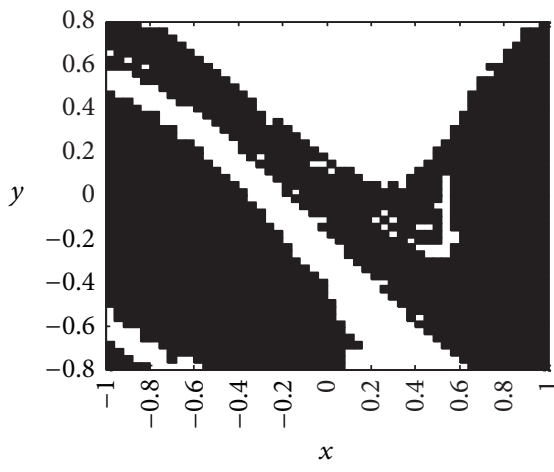

(c) $f_{1}=2.0, f_{2}=2.0, p=0.6368$

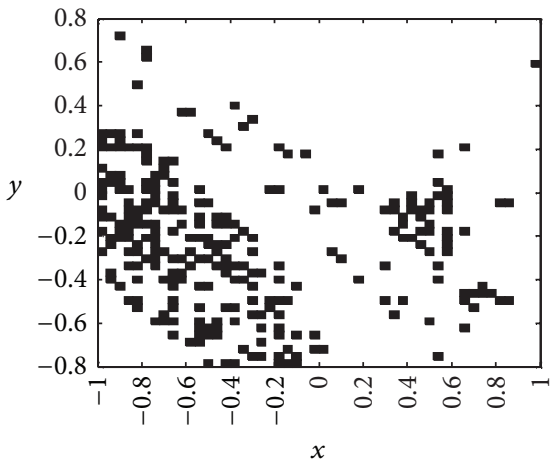

(f) $f_{1}=3.3, f_{2}=2.0, p=0.1056$

Figure 8: Erosion of safe basins in system (1): $\left(f_{2}=2.0\right)$.

the case when $f_{1} \leq f_{s}$, the boundary of the safe basins of system (1) are smooth; when $f_{s}<f_{1} \leq f_{e}$ the safe basins are eroded and when $f_{1}>f_{e}$, the safe basins disappear.

When $f_{2}=0$, the threshold value form condition (10) is $f_{1}=4.93$, which is close to the second safe basins bifurcation point $f_{e}=4.54$; it shows that there are strong relations between the erosion of safe basin and chaotic motions in the deterministic system.

3.2. Bifurcation with Bounded Noises. Next, we consider the effect of the random noise on the safe basins, the parameters in system (1) are chosen as

$$
\begin{array}{cccc}
\alpha=0.8, & \beta=0.9, & \varepsilon=0.1, & \rho=1.8, \\
\omega_{1}=1.5, & \omega_{2}=1.0, & \sigma=0.4, & f_{2}=2.0,
\end{array}
$$

which are the same as in deterministic case for comparison except that $f_{2}$ changes from 0 to 2 . Then, the erosion of the safe basin of system (1) is discussed numerically. For the method of numerical simulation, the reader is referred to Shinozuka and Jan [27], and the method of Monte-Carlo is used to generate random samples. Here, only 10 random samples are used in this paper due to the limitation of calculation capacity. If the solution of system (1) stays in area $D$ for a long enough time $t$ not less than 2000 in all the 10 random samples, such a solution can be approximately taken as a safe solution, and then the corresponding lattice may be taken as a part of the safe basins, which is defined in a similar way as for the deterministic one; if the solution of system (1) escapes from area $D$, such a solution is taken as an unsafe solution, and the corresponding lattice is not belonging to the safe basins. 
One may call such safe basins as stochastic safe basins. The governing equation (1) is numerically integrated by the fourth order Runge-Kutta algorithm, and the numerical results are shown in Figure 8(a) to Figure 8(g).

Figures $8(\mathrm{a})-8(\mathrm{~g})$ show that the stochastic safe basins are eroded more and more with increase of $f_{1}$, which is similar to the deterministic case as shown in Figures 7(a)-7(i), yet with significant differences in $f_{s}$ and $f_{e}$. Calculation results show that, in the case when $f_{1}>f_{s}=0.00$, the boundary of the stochastic safe basins begins to be eroded more and more with increase of $f_{1}$ as shown in Figure 8(b) to Figure 8(g) and, in the case when $f_{1}>f_{e}=3.43$, the stochastic safe basins disappear completely. One may call such phenomena that happen in the sudden change of the stochastic safe basins, which become from integrated one to eroded one or from eroded one to nothingness when $f_{1}$ passes through the critical values $f_{s}=0.00$ and $f_{e}=3.43$, as stochastic safe basins bifurcation. Then $f_{s}=0.00$ and $f_{e}=3.43$ are two bifurcation points if $f_{1}$ is chosen as bifurcation parameter. In the case when $f_{1} \leq f_{s}$, the boundary of the stochastic safe basins of system (1) are smooth; when $f_{s}<f_{1} \leq f_{e}$ the stochastic safe basins are eroded and when $f_{1}>f_{e}$, the safe basins disappear. The random disturbance $f_{2} \xi(t)$ causes $f_{s}$ and $f_{e}$ decrease from $f_{s}=0.21, f_{e}=4.54$ to $f_{s}=0.00$, $f_{e}=3.43$ and makes the system more unsafe in comparison with the deterministic case.

When $f_{2}=2.0$, the threshold value form condition (10) is $f_{1}=3.46$, which is close to the second safe basins bifurcation point $f_{e}=3.43$; it also shows that there are strong relations between the erosion of safe basin and chaotic motions in stochastic dynamical system, which is similar in the deterministic system. The incursive fractal fingers are also observed in the stochastic system, which means that chaotic responses still exist in the stochastic system (1) when $f_{2} \neq 0$. From Figure 8, one knows that random noise can aggravate the erosion of the safe basin.

Overall, random noise may destroy the integrity of the safe basins boundary, bring forward the occurrence of the stochastic bifurcation, and hence make the system become more unsafely. The threshold value of the stochastic dynamical system form condition (10) is $f_{1}=3.46$, which is smaller than the threshold value $f_{1}=4.93$ of the deterministic dynamical system. Obviously, random noises make the parametric threshold for chaotic motions vary in a larger region, hence making the chaotic motions occur more easily.

\section{Conclusions and Discussion}

The erosion of the safe basins and chaotic motions of a nonlinear vibroimpact oscillator under harmonic and bounded random noise is studied. Melnikov's method in the deterministic vibroimpact system is extended to the analysis of homoclinic bifurcations and chaos in the stochastic case. The results reveal that the threshold amplitude $f_{1}$ for the onset of chaos decreases as the noise amplitude $f_{2}$ increase.

Although the theory of stochastic bifurcation has been advanced to a new level in the last decade, there remain a lot of problems to be solved. Even the definition of stochastic bifurcation needs to be improved. In this paper, we suggest an alternative definition for stochastic bifurcation based on the analysis of the safe basins of a softening Duffing oscillator subject to deterministic harmonic and bounded random excitations, which focuses on a sudden change in the character of the safe basins of the dynamical system as the bifurcation parameter passes through a critical value. This definition applies equally well either to the stochastic bifurcation or to the deterministic bifurcation. However, the application of the definition for real systems needs more effect. The analysis shows that the random noise causes the two bifurcation points $f_{s}$ and $f_{e}$ decrease; the parametric threshold for chaotic motions varies in a larger region, therefore making the system more unsafely and making the chaotic motions occur more easily. From physical point of view, the results of this paper can help one to better design the system, such that the system operates in a nonchaotic state and then can be controlled more easily, meanwhile reducing the erosion of the safe basins which makes the system more secure.

In the paper, Melnikov's methods and bifurcation of safe basins are the main research methods. In the fact, there are other effective method to verify the chaos, for instance, topological horseshoes method which has been successfully applied in many works [28-30].

\section{Conflict of Interests}

The authors declare that there is no conflict of interests regarding the publication of this paper.

\section{Acknowledgments}

The work reported in this paper was supported by the National Natural Science Foundation of China under Grant no. 11401096 and 11326123, the Natural Science Foundation of Guangdong Province under Grant no. S2013010014485 and S201310012463, and Special fund of the Guangdong College discipline construction under Grant nos. 2013KJCX0189 and 2013B020314020.

\section{References}

[1] B. Brogliato, Non-smooth Mechanics: Models, Dynamics and Control, Springer, London, UK, 1999.

[2] V. S. Metrikyn, "On the theory of vibro-impact devices with randomly varying parameters," Izvestiya Vysshikh Uchebnykh Zavedenii, Radiofizika, vol. 13, pp. 4-12, 1970 (Russian).

[3] R. L. Stratonovich, Topics in the Theory of Random Noise, vol. 1-2, Gordon and Breach, New York, NY, USA, 1963.

[4] H. T. Zhu, "Stochastic response of vibro-impact Duffing oscillators under external and parametric Gaussian white noises," Journal of Sound and Vibration, vol. 333, no. 3, pp. 954-961, 2014.

[5] H.-S. Jing and M. Young, "Random response of a single-degreeof-freedom vibro-impact system with clearance," Earthquake Engineering \& Structural Dynamics, vol. 19, no. 6, pp. 789-798, 1990.

[6] M. Xu, Y. Wang, X. L. Jin, Z. L. Huang, and T. X. Yu, "Random response of vibro-impact systems with inelastic contact," International Journal of Non-Linear Mechanics, vol. 52, pp. 2631, 2013. 
[7] C. Li, W. Xu, J. Feng, and L. Wang, "Response probability density functions of Duffing-Van der Pol vibro-impact system under correlated Gaussian white noise excitations," Physica A, vol. 392, no. 6, pp. 1269-1279, 2013.

[8] V. F. Zhuravlev, "A method for analyzing vibration-impact systems by means of special functions," Mechanics of Solids, vol. 11, pp. 23-27, 1976.

[9] D. V. Iourtchenko and M. F. Dimentberg, "Energy balance for random vibrations of piecewise-conservative systems," Journal of Sound and Vibration, vol. 248, no. 5, pp. 913-923, 2001.

[10] Q. Feng and H. He, "Modeling of the mean Poincaré map on a class of random impact oscillators," European Journal of Mechanics, A: Solids, vol. 22, no. 2, pp. 267-281, 2003.

[11] D. V. Iourtchenko and L. L. Song, "Numerical investigation of a response probability density function of stochastic vibroimpact systems with inelastic impacts," International Journal of NonLinear Mechanics, vol. 41, no. 3, pp. 447-455, 2006.

[12] M. F. Dimentberg, O. Gaidai, and A. Naess, "Random vibrations with strongly inelastic impacts: response PDF by the path integration method," International Journal of Non-Linear Mechanics, vol. 44, no. 7, pp. 791-796, 2009.

[13] M. F. Dimentberg and D. V. Iourtchenko, "Random vibrations with impacts: a review, Nonlinear Dynamics, vol. 36, no. 2-4, pp. 229-254, 2004.

[14] J. Guckenherner and P. Holmes, "Nonlinear oscillations," in Dynamical Systems and Bifurcations of Vector Fields, Springer, New York, NY, USA, 1983.

[15] S.-N. Chow and S. W. Shaw, "Bifurcations of subharmonics," Journal of Differential Equations, vol. 65, no. 3, pp. 304-320, 1986.

[16] S. W. Shaw and R. H. Rand, "The transition to chaos in a simple mechanical system," International Journal of Non-Linear Mechanics, vol. 24, no. 1, pp. 41-56, 1989.

[17] Z. Du and W. Zhang, "Melnikov method for homoclinic bifurcation in nonlinear impact oscillators," Computers and Mathematics with Applications, vol. 50, no. 3-4, pp. 445-458, 2005.

[18] R. Stratonovich, Topics in the Theory of Random Noise, vol. 1, Gordon and Breach Science Publishers, New York, NY, USA, 1963.

[19] Y. K. Lin and G. Q. Cai, Probabilistic Structural Dynamics: Advanced Theory and Applications, Mc-Graw-Hill, New York, NY, USA, 1995.

[20] M. F. Dimentberg, D. V. Iourtchenko, and O. van Ewijk, "Subharmonic response of a quasi-isochronous vibroimpact system to a randomly disordered periodic excitation," Nonlinear Dynamics, vol. 17, no. 2, pp. 173-186, 1998.

[21] H. Rong, X. Wang, W. Xu, and T. Fang, "Subharmonic response of a single-degree-of-freedom nonlinear vibroimpact system to a randomly disordered periodic excitation," Journal of Sound and Vibration, vol. 327, no. 1-2, pp. 173-182, 2009.

[22] E. Simiu, Chaotic Transitions in Deterministic and Stochastic Systems, Princeton Series in Applied Mathematics, Princeton University Press, Princeton, NJ, USA, 2002.

[23] A. Wolf, J. B. Swift, H. L. Swinney, and J. A. Vastano, "Determining Lyapunov exponents from a time series," Physica D, vol. 16, no. 3, pp. 285-317, 1985.

[24] H. Rong, X. Wang, W. Xu, and T. Fang, "Resonant response of a non-linear vibro-impact system to combined deterministic harmonic and random excitations," International Journal of Non-Linear Mechanics, vol. 45, no. 5, pp. 474-481, 2010.
[25] R. P. Eason, A. J. Dick, and S. Nagarajaiah, "Numerical investigation of coexisting high and low amplitude responses and safe basin erosion for a coupled linear oscillator and nonlinear absorber system," Journal of Sound and Vibration, vol. 333, no. 15, pp. 3490-3504, 2014.

[26] C. B. Gan, "Noise-induced chaos and basin erosion in softening Duffing oscillator," Chaos, Solitons and Fractals, vol. 25, no. 5, pp. 1069-1081, 2005.

[27] M. Shinozuka and C.-M. Jan, "Digital simulation of random processes and its applications," Journal of Sound and Vibration, vol. 25, no. 1, pp. 111-128, 1972.

[28] X.-S. Yang, "Topological horseshoes and computer assisted verification of chaotic dynamics," International Journal of Bifurcation and Chaos, vol. 19, no. 4, pp. 1127-1145, 2009.

[29] Q. Li and X.-S. Yang, "A simple method for finding topological horseshoes," International Journal of Bifurcation and Chaos, vol. 20, no. 2, pp. 467-478, 2010.

[30] Q. Li, S. Tang, and X.-S. Yang, "New bifurcations in the simplest passive walking model," Chaos, vol. 23, no. 4, Article ID 043110, 2013. 


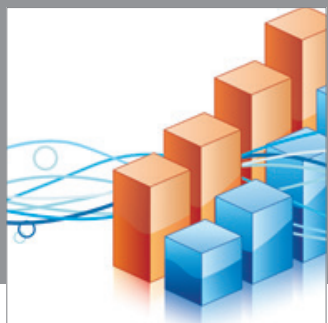

Advances in

Operations Research

mansans

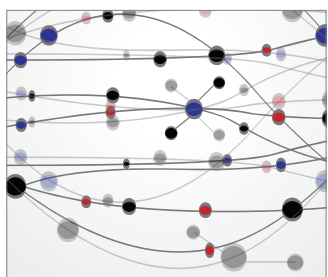

The Scientific World Journal
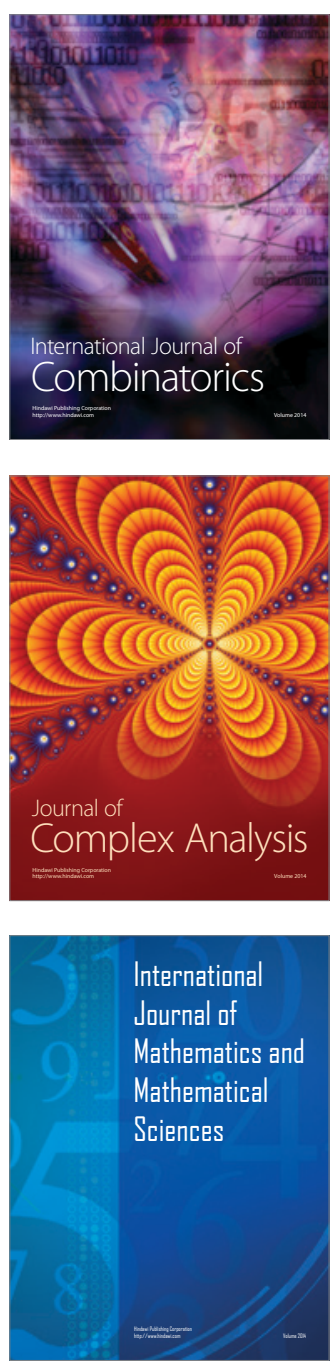
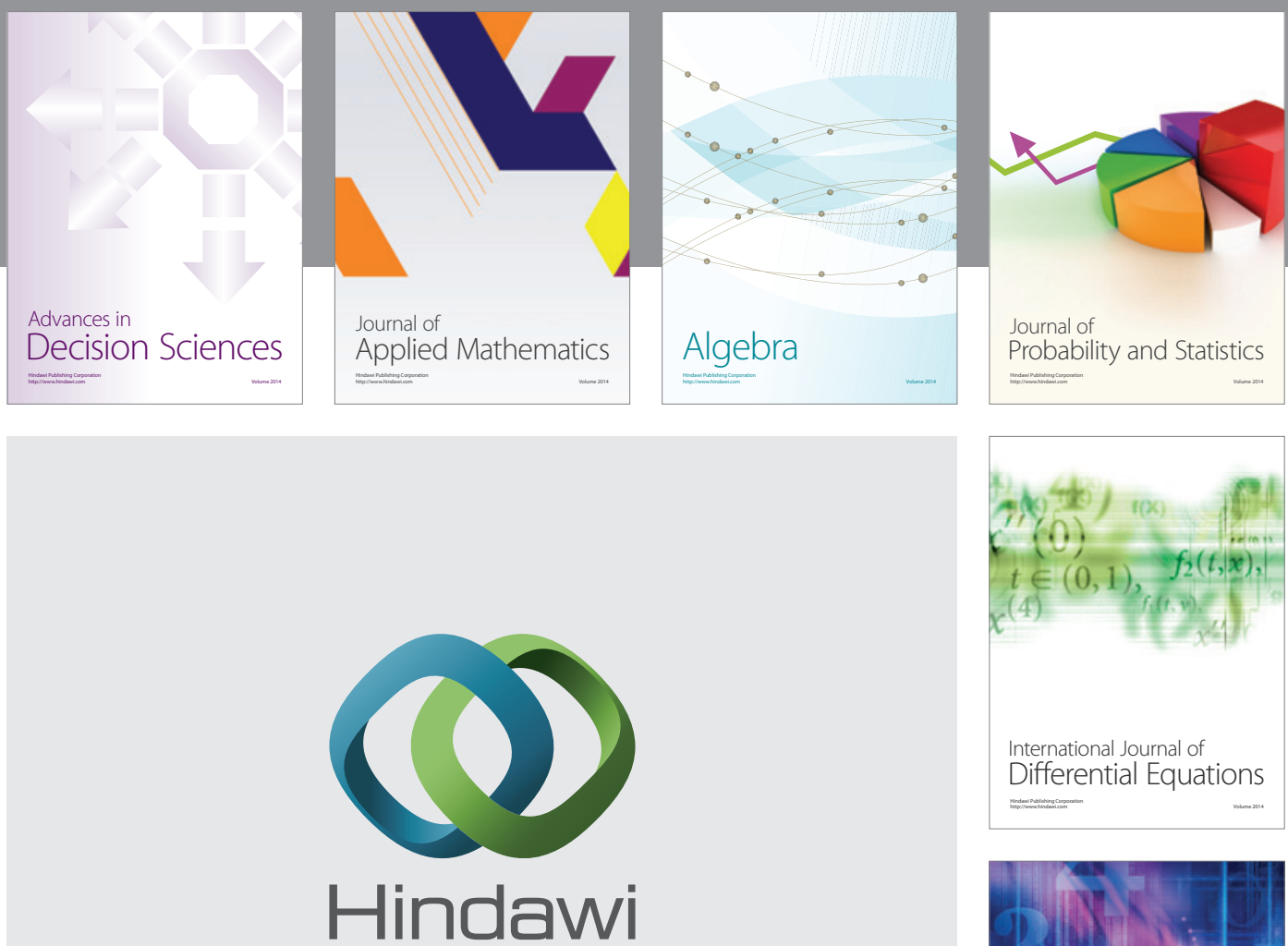

Submit your manuscripts at http://www.hindawi.com
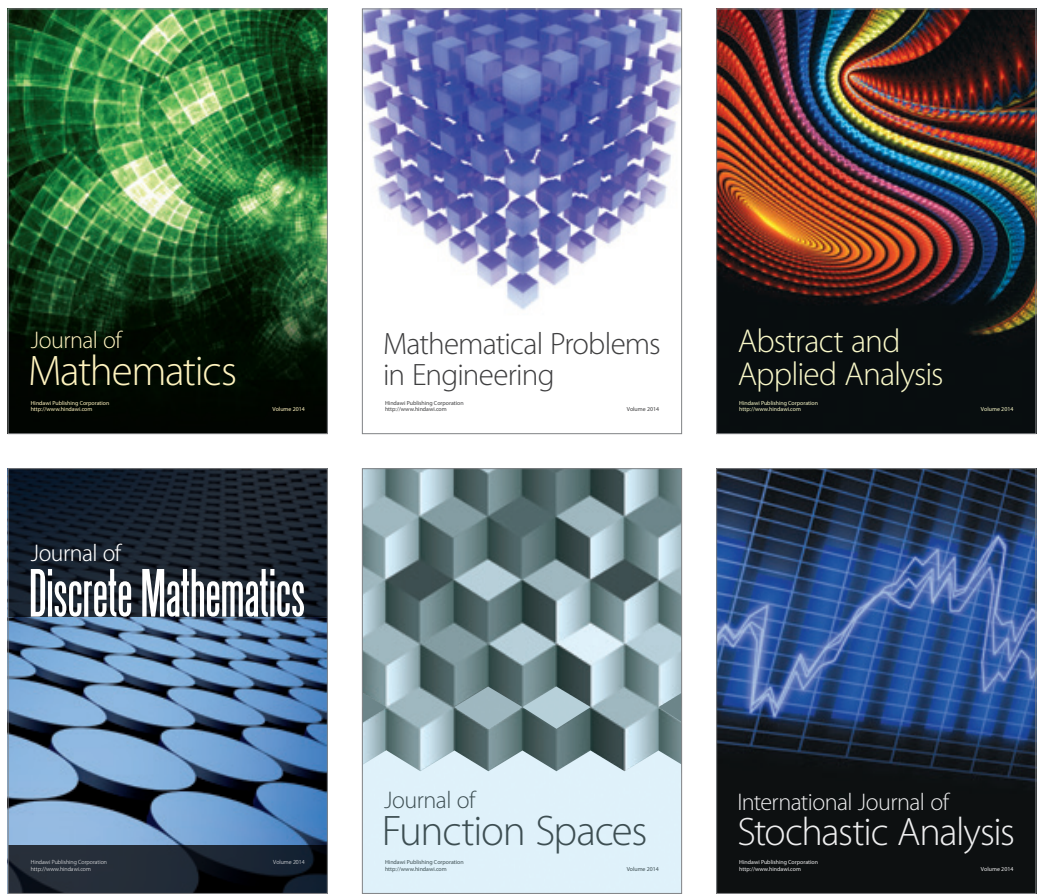

Journal of

Function Spaces

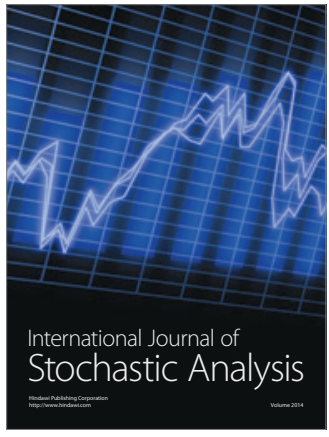

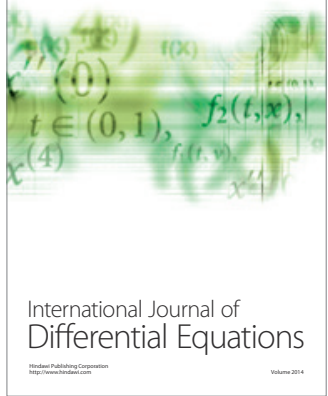
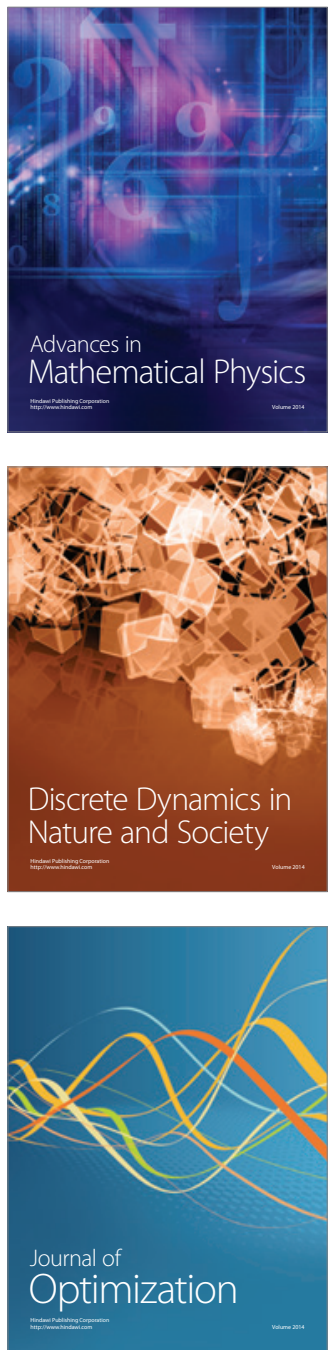